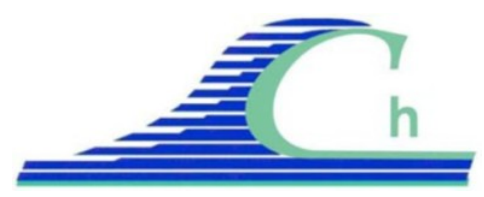

XII ${ }^{\text {ìmes }}$ Journées Nationales Génie Côtier - Génie Civil

Cherbourg, 12-14 juin 2012

DOI:10.5150/jngcgc.2012.108-B @ Editions Paralia CFL

disponible en ligne - http://www.paralia.fr - available online

\title{
Caractérisation rhéologique des sédiments de dragage
}

\author{
Ahmed BENAMAR ${ }^{1}$, Sébastien BRASSELET ${ }^{2}$, Anne PANTET ${ }^{1}$ \\ 1. Université du Havre, LOMC, UMR 6294, CNRS-Université du Havre, France. \\ 2. Grand Port Maritime du Havre, service A.E.M., Le Havre, France. \\ anne.pantet@univ-lehavre.fr
}

\section{Résumé :}

Les pratiques de dragage constituent un enjeu pour le développement et le maintien des activités portuaires et fluviales. Elles présentent également un risque de contamination des eaux côtières pouvant affecter durablement les écosystèmes. La réglementation française et européenne prévoit que les vases polluées ne peuvent plus être rejetées dans le milieu marin obligeant à terme les collectivités à prévoir un traitement à terre coûteux en raison des volumes considérables à valoriser et de la complexité d'un tel processus. Un recyclage ou une valorisation sont d'autant plus importants que ce sont des millions de mètres cubes de sédiments qui doivent être extraits des ports européens.

Le travail présenté ici porte sur l'analyse du comportement rhéologique des sédiments en fonction de la teneur en eau (ou la concentration) et également de la teneur de la fraction granulaire. Les propriétés rhéologiques des sédiments ont été mesurées (seuil de cisaillement, viscosité) avec différentes géométries (plan-plan et cylindres coaxiaux) à l'aide d'un rhéomètre. Cette étude permettra de déterminer les domaines d'application des outils en fonction des constituants sédimentaires, fins à grossiers. Par ailleurs, elle pourra orienter le maître d'ouvrage sur les dispositions techniques à adopter sur le terrain de façon à améliorer la navigabilité en présence des sédiments.

Ce travail est mené en collaboration avec le GPMH et rentre dans le cadre du projet RESSOLV du GRR Sciences de l'Environnement et Risques de la région Haute Normandie.

\section{Mots-clés :}

Sédiments - Travaux maritimes - Gestion portuaire - Environnement littoral Ecosystèmes côtiers - Valorisation dans la construction - Navigabilité des navires

\section{Introduction}

Le Grand Port Maritime du Havre (GPMH) drague chaque année environ 3 millions de $\mathrm{m}^{3}$ de sédiments qui sont principalement clapés en mer et exceptionnellement déposés à terre (chambre de dépôt de Tancarville). Les navires étant de plus en plus conséquents en taille et en capacité de stockage (portes conteneurs, vraquiers, rouliers, ...), leur tirant d'eau devenant de plus en plus important, il faut donc prévoir une plus grande profondeur des chenaux d'accès et des bassins. Durant l'année 2007, près de $60000 \mathrm{~m}^{3}$ 
de sédiments issus des dragages de la darse en aval de l'écluse du canal de Tancarville ont été mis à terre dans une chambre de dépôt aménagée à cet effet. Cette gestion des matériaux à terre est un choix économique fait par le GPMH et non une obligation réglementaire.

L’objectif de ce travail est de caractériser les propriétés rhéologiques des vases du Port du Havre dans le but notamment de les valoriser dans des coulis de comblement de cavités. Pour l'ensemble des essais rhéologiques effectués, les matériaux utilisés ont été prélevés dans la partie amont de la nouvelle écluse de Tancarville. La caractérisation des sédiments présentée est générale, et s’intéresse principalement aux effets de la concentration initiale et de l'ajout de matières à valoriser (cendres volantes). Elle a pour but d'examiner deux axes de recherche appliquée pour réduire le tonnage de matériaux dragués et éventuellement stockés à terre.

Le premier axe consiste à réduire le volume de matériaux à draguer. Pour les ports à taux d'envasement élevé, la préoccupation de garantir le passage en toute sécurité des navires implique des coûts d'entretien importants, qui augmentent avec la capacité des navires. Dans cette perspective, le concept de fond marin navigable a été développé ; il s'agit de remanier le sédiment par agitation et permettre en outre de garantir une profondeur navigable sans avoir à effectuer des travaux de dragage.

Le second axe de recherche envisage l'utilisation de ces matériaux stockés dans les filières de production de matériaux de construction. Il existe actuellement des entreprises utilisant des sédiments marins dans la composition de matériaux, tels que HZD en Allemagne dans la fabrication de briques et VBC 3000 en France dans la fabrication de granulats d'argiles expansées et de briques.

Ainsi est-il nécessaire dans des processus industriels d'avoir une évaluation des possibilités de pompage et de mise en œuvre à l'extrusion de briques.

Dans les deux cas d'étude, avoir une caractérisation mécanique du sédiment à différentes consistances (de l'état fluide à pâteux) est une donnée essentielle. Ainsi la transition d'état dans les sédiments cohésifs (BERLAMONT et al., 1993 ; PANTET \& MONNET, 2007) peut être liée à la variation de la teneur en eau (i.e. la concentration) mais également à la structuration des particules, qui fait intervenir la notion de seuil d'écoulement ou de rigidité, permettant de distinguer un comportement solide et un comportement fluide en fonction de l'état de contrainte appliqué. Cette structuration est aussi fortement dépendante du temps, indiquant le caractère thixotrope des matériaux. La nature et la taille des particules fines et la physico-chimie de leur environnement liquide sont des paramètres importants dans la caractérisation de la structuration. Cette notion de seuil est complexe et même remise en question (BARNES \& WALTERS, 1985 ; BARNES, 1999 ; COUSSOT et al., 2009). En effet, avec le développement d'appareils de plus en plus performants, ce passage solide-liquide en fonction du gradient de vitesse n'est plus aujourd'hui aussi net que celui défini initialement avec les fluides non-newtoniens. Si l'existence d'un ou de plusieurs seuils de contrainte de 


\section{XII ${ }^{\text {èmes }}$ Journées Nationales Génie Côtier - Génie Civil \\ Cherbourg, 12-14 juin 2012}

cisaillement est désormais acceptée, il doit être associé à un domaine de taux de cisaillement et à un état structurel de référence.

\section{Matériels et méthodes}

\subsection{Les vases}

Le matériau a été prélevé le 11/05/2011 à l'aide d'une pelle à treillis munie d'une benne preneuse. Puis, les échantillons ont été stockés dans des récipients étanches et placés dans une enceinte réfrigérée à $6^{\circ} \mathrm{C}$ afin de conserver leur teneur en eau initiale (de l'ordre de 103\%) et éviter aussi leur évolution. Les valeurs de bleu de méthylène (NF P 94-068) mesurées sur six échantillons varient de 2,1 à 3,3 et les limites de liquidité et de plasticité sont voisines, respectivement, de $94 \%$ et $39 \%$. Ces valeurs indiquent des sols limoneux de plasticité élevée suivant le GTR 92. Les paramètres granulométriques des échantillons testés au granulomètre laser (Multisizer 2000 - Malvern) indiquent une fraction sableuse de l'ordre de 10 à $14 \%$, des fractions de passant $d_{10}=3 \mu \mathrm{m}$ et $\mathrm{d}_{90}=60 \mu \mathrm{m}$ et un coefficient d'uniformité $\mathrm{Cu}$ entre 4,20 et 4,80. Les essais de consolidation ont permis de déterminer un indice de compressibilité Cc compris entre 0,96 et 1,37. La teneur en sels a été mesurée avec une cellule conductimétrique étalonnée au préalable en fonction de la concentration en $\mathrm{NaCl}$. Elle peut être estimée entre 26 et 31 g/l.

\subsection{Techniques rhéométriques}

La caractérisation rhéologique des sédiments a été menée à l'aide d'un rhéomètre Anton Paar de type MCR 501, nouvellement installé au laboratoire. Les essais rhéologiques ont été effectués avec une géométrie plan-plan (diamètre $50 \mathrm{~mm}$ ) et avec une géométrie cylindrique coaxiale (diamètre du rotor $27 \mathrm{~mm}$, et diamètre du godet $29 \mathrm{~mm}$ ), en vitesses imposées ou en contraintes imposées. Les géométries présentent un état de surface rugueux. Le but étant de comparer les résultats obtenus avec les deux géométries et de discuter le choix de la définition de la valeur seuil de contrainte.

Le protocole retenu en vitesse de cisaillement imposée est décomposé en quatre phases : - pré-cisaillement du matériau afin de l'homogénéiser à $30 \mathrm{~s}^{-1}$ pendant $30 \mathrm{~s}$, - repos de $30 \mathrm{~s}$,

- démarrage de l'essai, augmentation constante de la vitesse de 0 à $200 \mathrm{~s}^{-1}$ en $12 \mathrm{~min}$, - réduction constante de la vitesse imposée en 12 min jusqu'à la fin de l'essai.

Les systèmes de mesure couramment utilisés présentent des inconvénients et ceux utilisés dans cette étude n'échappent pas à la règle (effet de paroi, représentativité des échantillons, taille des grains, vibration, ...). La rhéologie bien que très tributaire des modes opératoires permet d'obtenir des informations quantitatives de l'état du matériau. Plusieurs tests préliminaires ont été effectués pour établir le protocole qui garantisse une bonne comparaison des résultats obtenus en fonction des conditions de préparation, notamment l'effet du malaxage et du temps d'aération (oxygénation des sédiments). 
Compte tenu des remarques présentées dans l'introduction, concernant la définition du seuil d'écoulement, nous définirons la rigidité initiale du sédiment comme la valeur de la contrainte de cisaillement à $10^{-2} \mathrm{~s}^{-1}$ sur la courbe de montée et ce, malgré l'existence d'un minimum local en contrainte entre 0,1 et $3 \mathrm{~s}^{-1}$ et d'un seuil obtenu par extrapolation de la courbe en descente aux très faibles vitesses de cisaillement nettement plus faible. Nous nous limiterons à comparer ces valeurs retenues. Les figures $1 \mathrm{a}$ et $1 \mathrm{~b}$ montrent l'effet de la durée du malaxage à $160 \mathrm{tr} / \mathrm{min}$ sur la vase avec un agitateur à ailettes. Il est à noter une réelle modification des matériaux avec le malaxage même court (10 s). L'exposition à l'air des vases a également une influence nette sur les propriétés rhéologiques des vases. Une différence est notée entre l'état initial et l'état après $1 \mathrm{~h}$ d'exposition à l'air, mais ce dernier n'évolue pas malgré 2 h d'exposition supplémentaire. La concentration initiale du sédiment étant voisine de $670 \mathrm{~g} / \mathrm{l}$ (teneur en eau voisine de 150\%), les échantillons sont malaxés et dilués en ajoutant de l'eau provenant d'un bassin du port et les essais rhéologiques sont réalisés dans la demijournée qui suit. Ces durées sont donc compatibles avec les essais. A ce stade de l'étude, ce protocole de préparation sera adopté afin d’obtenir une comparaison des essais compte tenu de l'évolution possible de la matière organique des vases. Toutefois, il reste à mieux le détailler pour expliquer ces changements nets entre la vase à l'état naturel et l'effet combiné du malaxage et de l'oxygénation.
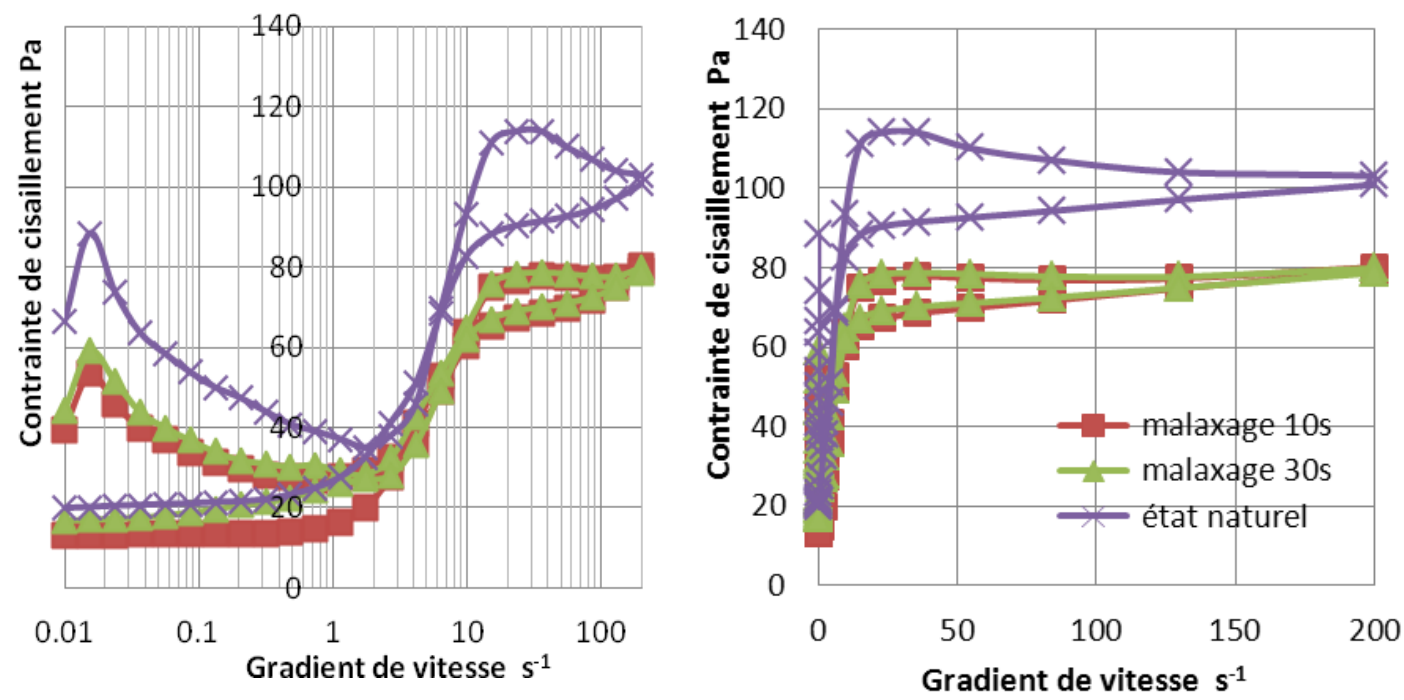

Figure 1. Courbes d'écoulement en mode vitesses imposées de la vase de Tancarville avec une géométrie plan-plan à deux échelles - effet du temps de malaxage

\section{Résultats}

Les courbes expérimentales sont présentées en fonction de la concentration sur les figures 2 et 3 pour chaque géométrie en vitesses imposées. Pour les deux systèmes, les courbes expérimentales présentent des allures similaires avec un comportement 


\section{XII ${ }^{\text {èmes }}$ Journées Nationales Génie Côtier - Génie Civil \\ Cherbourg, 12-14 juin 2012}

complexe entre 0,01 et $1 \mathrm{~s}^{-1}$, indiquant l'existence d'un minimum local en contrainte pour toutes les concentrations lors de la montée en vitesse, qui n’apparait pas lors de la décroissance. Ce comportement est souvent observé et est lié à des mécanismes de structuration-déstructuration et de thixotropie dans les matériaux argileux (BESQ et al., 2003), cimentaires (ROUSSEL, 2005) et vaseux (PANTET et al., 2010).

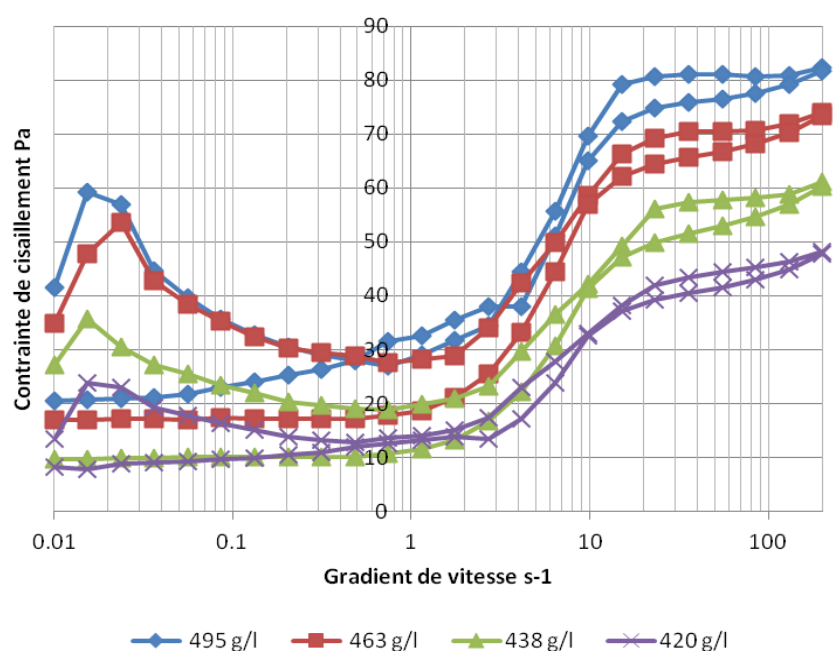

Figure 2. Courbes d'écoulement en mode vitesses imposées de la vase de Tancarville à différentes concentrations avec une géométrie plan-plan.

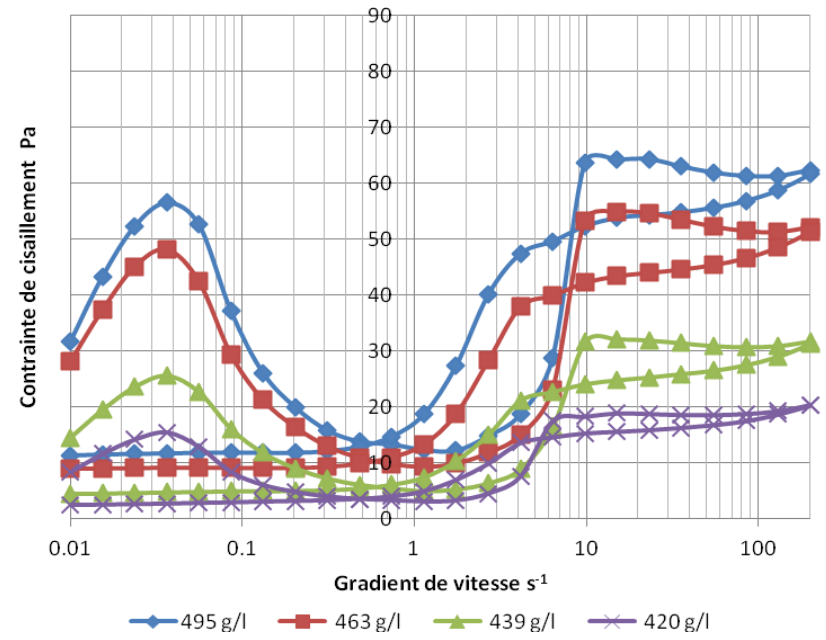

Figure 3. Courbes d'écoulement en mode vitesses imposées de la vase de Tancarville à différentes concentrations avec une géométrie cylindrique.

En montée, la relation entre le gradient de vitesse et la contrainte est non monotone donnant lieu à un écoulement en bandes de cisaillement ou shear bands, (comme dans les sols). Dans les systèmes colloïdaux, il est possible d'obtenir sous écoulement la coexistence d'une phase solide (peu ou pas cisaillée) et d'une phase liquide sous une contrainte de cisaillement comparable. Seules des mesures locales dans l'écoulement 
(vélocimétrie par images de particules PIV, vélocimétrie ultra-sonore, IRM) peuvent le confirmer. A cause de cette localisation du cisaillement, les grandeurs rhéologiques mesurées dans ce domaine ne sont que des grandeurs apparentes sur un matériau considéré homogène. Avec la géométrie cylindrique, un plateau s’établit dans la phase de vitesse décroissante, dès $2 \mathrm{~s}^{-1}$, alors qu'avec la géométrie à disques, la relation est linéaire. A titre de comparaison, les valeurs de seuil initial à $10^{-2} \mathrm{~s}^{-1}$ sont rassemblées dans le tableau 1 en fonction de la concentration. Pour chaque type d'essai, on note une organisation en fonction de la concentration suivant une loi classique exponentielle, souvent proposée en première approche. Pour la partie expérimentale détaillée (essais à vitesse imposée en géométrie plan-plan), la relation exponentielle s'exprime par $\left.y=0,26 \times e^{0,0104 . x}\right)$, y étant la valeur de la rigidité initiale (définie sur la courbe de montée) et $\mathrm{x}$ la concentration en matière solide (exprimée en $\mathrm{g} / \mathrm{l}$ ).

Tableau 1. Synthèse des données relatives à l'étude rhéologique.

\begin{tabular}{|c|c|c|c|c|}
\hline \multirow{2}{*}{$\begin{array}{l}\text { Teneur } \\
\text { en eau \% }\end{array}$} & \multirow{2}{*}{$\begin{array}{l}\text { Concentration } \\
(\mathrm{g} / \mathrm{l})\end{array}$} & \multicolumn{2}{|c|}{ Vitesses imposées } & \multirow{2}{*}{$\begin{array}{l}\text { * Contraintes imposées } \\
\text { Seuil initial (Pa) } \\
\text { Géométrie plan-plan }\end{array}$} \\
\hline & & $\begin{array}{l}\text { Seuil initial } \\
\text { montée }(\mathrm{Pa}) \\
\text { Géo. plan-plan }\end{array}$ & $\begin{array}{l}\text { Seuil initial } \\
\text { montée }(\mathrm{Pa}) \\
\text { Géo. cylindres }\end{array}$ & \\
\hline 159,2 & 506 & 51 & & 36 \\
\hline 164,1 & 494 & 41 & 32 & 32 \\
\hline 177,5 & 463 & 36 & 28 & 20 \\
\hline 188,9 & 460 & 35 & & \\
\hline 189,2 & 438 & 27 & 15 & 13 \\
\hline 199,2 & 420 & 14 & 8 & 5 \\
\hline 248,1 & 350 & 11 & & 4 \\
\hline
\end{tabular}

*Pour mémoire, les essais réalisés en contrainte imposée sont conformes à ceux réalisés par un autre laboratoire (GREISER, 2009) en utilisant le même protocole

Cependant, ces valeurs spécifiques ainsi que les courbes présentent des écarts significatifs en fonction de la géométrie. Dans les deux configurations, les effets de bord sont négligeables compte tenu des dimensions. Avec la géométrie cylindrique, le cisaillement interne varie en fonction de la distance à l'axe central. Avec les disques coaxiaux, le gradient de vitesse est nul près de l'axe et maximal en périphérie, l'état de structuration de fluide peut être inhomogène dans l'échantillon. Les conditions pratiques de réalisation des essais sont plus simples avec la géométrie plan-plan. Il est difficile de donner une conclusion définitive sur le choix des géométries avec cette étude de base.

L'autre axe de valorisation des sédiments est leur utilisation dans la composition de matériaux de construction. Pour des raisons techniques, les essais ont été réalisés en géométrie plan-plan. Afin de mettre en évidence l'effet d'ajouts sur le comportement des formulations à base de produits déchets de l'industrie, des cendres volantes ont été ajoutées à la vase naturelle par malaxage pour obtenir un coulis. On observe sur la 


\section{XII ${ }^{\text {èmes }}$ Journées Nationales Génie Côtier - Génie Civil \\ Cherbourg, 12-14 juin 2012}

figure 4 que leur apport ne modifie pas l'allure des courbes d'écoulement. La courbe d'écoulement en montée est toujours non monotone avec l'existence de plusieurs valeurs de taux de cisaillement compatibles avec une même contrainte de cisaillement. La courbe d'écoulement en descente est continûment décroissante. L'effet de l'apport de particules fines sur les trois formulations n'est pas uniforme, il s'accentue avec les plus fortes valeurs. Les courbes d'écoulement à l'état naturel et avec un ajout modéré (3\% et $5 \%$ - pourcentages massiques) se superposent au faible gradient de vitesse à la montée et à la descente. L'effet de l'ajout de la fraction granulaire fine reste ici limité, à cause des conditions de géométrie compatible avec la taille des grains les plus gros. D'autres systèmes de mesures permettent de suivre l'évolution des paramètres rhéologiques d'une suspension de vase naturelle additionnée de sables fins à grossiers (SCHATZMANN et al., 2009).

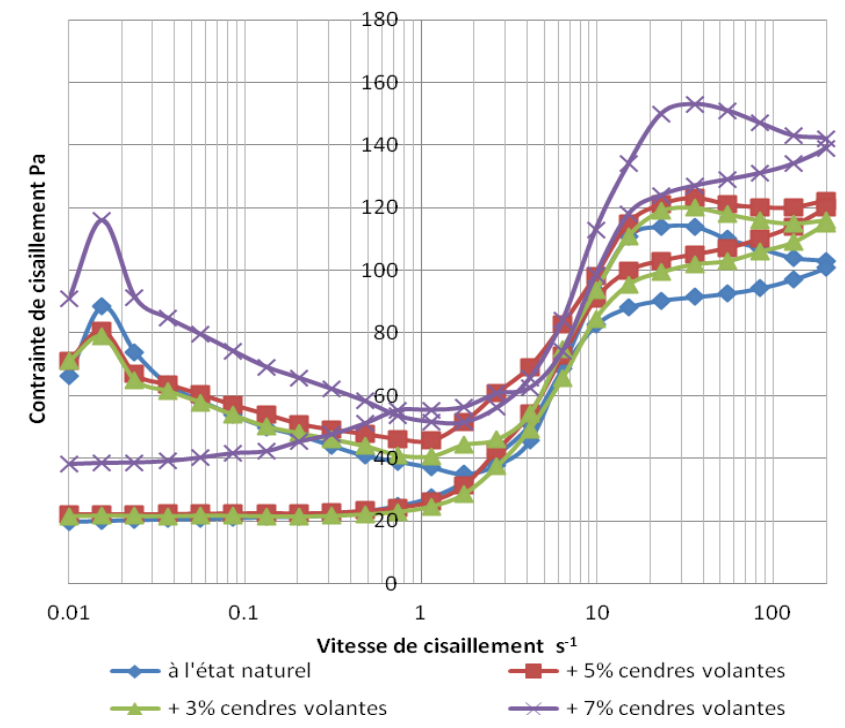

Figure 4. Courbes d'écoulement en mode vitesses imposées d'une formulation vasecendres volantes à différentes concentrations, analysées avec une géométrie plan-plan.

\section{Conclusions}

Des mesures expérimentales ont été effectuées pour caractériser les propriétés rhéologiques d'une vase naturelle à partir d'un rhéomètre de haute précision avec deux géométries. Les résultats expérimentaux montrent que les paramètres de comportement dépendent non seulement de la concentration mais aussi du gradient de vitesse et de la structuration. La forme non monotone de la courbe d'écoulement en montée quelle que soit la géométrie utilisée indique qu'il existe des niveaux de contrainte de cisaillement compatibles avec plusieurs mécanismes d'écoulement associés à des gradients de vitesse différents. En descente, les courbes d'écoulement sont continûment décroissantes, voire présentent un plateau. Les modèles empiriques classiques (Bingham, Herschel-Bulkley, ...) de détermination du seuil d'écoulement ne traduisent 
pas ces mécanismes locaux de structuration-déstructuration à l'échelle microscopique. Ces phénomènes font régulièrement l’objet de recherches fondamentales approfondies pour trouver leur application dans la pratique. Ainsi, dans le cadre de l'étude sur les fonds navigables, comme il est difficile de définir une valeur de contrainte seuil sans la rattacher au domaine de cisaillement considéré, identifier uniquement cette grandeur comme indicateur de navigabilité reste délicat. A la notion de seuil est associée également la viscosité du fluide, qui traduit le comportement du matériau à l'état fluide. Pour l'axe de valorisation des vases dans la composition de matériaux de construction, l'ajout d’une fraction plus grossière augmente modérément les caractéristiques initiales, toutefois il faut préciser que cet apport en particules non colloïdales doit être limité et rester compatible avec les conditions d'utilisation du dispositif employé.

\section{Références bibliographiques}

BARNES H.A., WALTERS K. (1985). The yield stress myth. Rheologica Acta, Vol. 24, Number 4, pp 323-326. doi:10.1007/BF01333960

BARNES H.A. (1999). The yield stress-a review or-everything flows. Journal of NonNewtonian Fluids Mechanics, Vol. 81(1-2), pp 133-178. doi:10.1016/S0377-0257(98)00094-9

BERLAMONT J., OCKENDEN M., TOORMAN E., WINTERWERP J. (1993). The characterization of cohesive sediments properties, Coastal Engineering, 21, pp 105-128. doi:10.1016/0378-3839(93)90047-C

BESQ A., MALFOY C., PANTET A., MONNET P., RIGHI D. (2003). Physicochemical characterization and flow properties of some bentonite muds. Applied Clay Science, Vol. 23, pp 275 -286. doi:10.1016/S0169-1317(03)00127-3

COUSSOT P., TOCQUER L., LANOS C., OVARLEZ G. (2009). Macroscopic vs.local rheology of yield stress fluids. J. of Non-Newtonian Fluid Mech., Vol. 158, pp 85-90. doi:10.1016/j.jnnfm.2008.08.003

GREISER N (2009). Rheological Measurements on Sediment Samples from Tancarville Barge Locks, Le Havre. Sediment Management Consulting, 10 p.

PANTET A., MONNET P. (2007). Liquid-solid transition of kaolinite suspensions. Mechanics of Materials, Vol. 39, Issue 9, pp 819-833. doi:10.1016/j.mechmat.2006.12.004 PANTET A, ROBERT S., JARNY S., KERVELLA S. (2010). Effect of Coarse Particle Volume Fraction on the Yield Stress of Muddy Sediments from Marennes Oléron Bay. Advances in Materials Science and Engineering Vol. 2010, Article ID 245398, 6 p.

ROUSSEL N. (2005). Steady and transient flow of fresh cement pastes. Cement and Concrete Research, Vol. 35, pp 1656-1664. doi:10.1016/j.cemconres.2004.08.001

SCHATZMANN M., BEZZOLA G. R., MINOR H.-E., WINDHAB E. J., FISHER P. (2009). Rheometry for large particulated fluids: analysis of the ball measuring system and comparison to debris flow rheometry. Rheologica acta, Vol. 48, n 7, pp 715-733. doi:10.1007/s00397-009-0364-x 